\section{Entre taux de rétention passif et taux de rétention actif : une analyse de la motivation à partir du MOOC « Découvrir la science politique » (Louv3x) sur la plateforme edX}

Comparing passive and active retention rates: An analysis of motivation in the MOOC "Introduction to Political Science" (Louv3x) on the Open edX platform
*Nathalie Schiffino nathalie.schiffino@uclouvain-mons.be

*Maximilien Cogels maximilien.cogels@uclouvain.be

*Pierre Baudewyns pierre.baudewyns@uclouvain.be

*Ella Hamonic ella.hamonic@uclouvain.be

*Vincent Legrand vincent.legrand@uclouvain.be

*Min Reuchamps min.reuchamps@uclouvain.be

*Université catholique de Louvain, BELGIQUE

Compte rendu d'expérience intégrant les TIC

\section{Résumé}

Une des critiques récurrentes à l'égard des MOOC porte sur le fait que peu d'apprenants terminent les cours auxquels ils se sont inscrits et obtiennent finalement un certificat. Cet article aborde la problématique à partir de l'analyse du MOOC « Découvrir la science politique » (dont l'acronyme est Louv3x et qui est disponible sur la plateforme edX) : avec un taux de rétention de $18 \%$, il fait figure d'exception. Pour appréhender empiriquement la problématique, tout en s'appuyant sur la littérature existante, les auteurs opèrent une distinction entre taux de rétention active et taux de rétention passive. Dans cette perspective, et sur la base des données quantitatives et qualitatives récoltées pendant la durée $\mathrm{du} \mathrm{MOOC}$, cette contribution explore les facteurs explicatifs de la rétention et de la motivation des apprenants et de l'équipe pédagogique.

\section{Mots-clés}

MOOC, Louv3x, edX, science politique, rétention active, rétention passive, motivation, engagement

\section{Abstract}

One recurring criticism about MOOCs is the fact that few learners complete the course they have registered for and finally earn a certificate. This article delves into this issue on the basis of the analysis of the MOOC «Découvrir la science politique» (Louv3x, on the edX platform). With a retention rate of $18 \%$, this MOOC scores above the average. In order to refine empirically the approach, and building on the existing literature, the authors distinguish between levels of active retention and passive retention. In this perspective, the paper digs into data collected throughout the MOOC and explores several explanatory factors of retention and motivation for the learners and the teaching team.

\section{Keywords}

MOOC, Louv3x, edX, Political Science, active retention, passive retention, motivation, engagement 


\section{Introduction}

Depuis 2007, des cours en ligne gratuits et destinés à un large public - connus sous l'acronyme MOOC (massive open online courses) - ont fait leur apparition en provenance de différents pays à travers le monde (Karsenti, 2013). Ce tournant pédagogique, qui n'est toutefois pas si récent (Gaebel, 2013), a notamment été amorcé par le MOOC de Siemens et Downes à l'Université du Manitoba en 2008 (Cassidy, Breakwell et Bailey, 2014; Toven-Lindsey, Rhoads et Berdan Lozano, 2015). Alors que le New York Times (Pappano, 2012) décrétait 2012 comme étant l'année des MOOC, les analyses les plus récentes pointent encore le manque de connaissances sur ce type de cours (Toven-Lindsey et al., 2015; Zheng, Rosson, Shih et Carroll, 2015). Perçus par certains observateurs comme un engagement des apprenants vis-à-vis du processus d'apprentissage (Cormier et Gillis, 2010), ils laissent d'autres analystes sceptiques quant aux modalités d'apprentissage et à la capacité critique qu'ils développent chez les apprenants (Allen et Seaman, 2013; Giroux, 2011). La production scientifique s'interroge notamment sur le taux de rétention et le lie à l'engagement des apprenants et des équipes pédagogiques (Cassidy et al., 2014; Cheung, 2014; Khalil et Ebner, 2014; Onah, Sinclair et Boyatt, 2014), ainsi qu'à leurs motivations (El-Hmoudova, 2014; Hew et Cheung, 2014) dans une perspective critique sur la qualité de l'apprentissage (Toven-Lindsey et al., 2015; Vallières, 2013).

Sur la base des chiffres des MOOC délivrés par les différentes plateformes (edX, Coursera, Udacity, etc.), les études montrent que seul un petit nombre d'apprenants inscrits à un MOOC le suivent jusqu'à son terme : certaines avancent des chiffres allant de 5 à $10 \%$ des apprenants (Anderson, 2013; Dillenbourg, Fox, Kirchner, Mitchell et Wirsing, 2014; Gaebel, 2013), d'autres mentionnent qu'il s'agirait de moins de $13 \%$ des apprenants (Onah et al., 2014, p. 5825). Avec un taux de rétention de $18 \%$, le MOOC « Découvrir la science politique »
$(\operatorname{Louv} 3 \mathrm{x})^{1}$ fait figure d'exception. Ce chiffre remet d'autant plus en question l'engagement et la motivation des apprenants, selon les termes de la littérature, que le certificat n'était octroyé qu'aux inscrits obtenant un pourcentage de réussite d'au moins $75 \%$. Cet article vise donc à contribuer, d'une part, aux études empiriques qui se développent dans le domaine de la pédagogie et des technologies universitaires, mais dont les données ne concernent pas encore l'enseignement de la science politique et, d'autre part, aux recherches sur la rétention, l'engagement et la motivation, ces notions étant par ailleurs souvent mobilisées sans être définies.

Afin de définir, d'analyser et d'expliquer le taux de rétention à partir du MOOC Louv3x, nous aborderons d'abord la double approche souvent employée (Fairhurst et Fairhurst, 1995) : d'abord, celle de l'équipe pédagogique et de la structure du cours, mais aussi ensuite, celle du profil type des apprenants et de leurs motivations et attentes, ainsi que l'évolution de cellesci avant et après le MOOC. Nous montrerons en quoi la rétention dans un MOOC est liée à l'engagement et/ou à la motivation à la fois de l'équipe pédagogique et des apprenants, en proposant une définition des notions.

En termes de méthodologie, notre analyse repose sur la base des données statistiques récoltées tout au long du MOOC, en particulier grâce à une enquête pré- et post-MOOC. Ainsi, avant et après le cours, un questionnaire a été envoyé aux apprenants inscrits. Le pré-questionnaire a été envoyé par courriel depuis le logiciel LimeSurvey à tous les apprenants qui étaient inscrits trois jours avant le début du cours, ainsi qu'à une partie des retardataires connus par la suite : au total, 6470 invitations ont été envoyées avec, au final, 1593 pré-questionnaires remplis. Le post-question-

1 En Belgique, c'est en février 2014 que les premiers cours MOOC ont été réalisés à l'Université catholique de Louvain (UCL). Quatre cours y ont été enseignés sur la plateforme edX, deux en langue française (Lou$v 3 x$ et Louv $4 x$ ) et deux en langue anglaise (Louv1x et Louv2x). À la suite de cette première expérience dont elle a été pionnière en Belgique, l'UCL a décidé d'augmenter son offre à 15 MOOC pour l'année académique 2014-2015. 
naire, quant à lui, a été envoyé depuis le même logiciel à 7667 apprenants (incluant donc les apprenants ayant continué à s'inscrire au MOOC après son démarrage), avec, au final, 1423 post-questionnaires remplis. De manière parallèle, nous avons archivé les commentaires reçus par courriel ou postés sur les forums du MOOC, ce qui nous permettra de compléter l'analyse de manière qualitative.

\section{1. Équipe pédagogique et structure du MOOC Louv3x}

\subsection{La nécessaire cohésion d'une équipe pluridisciplinaire}

Selon Adamopoulos, le professeur, son équipe et, plus généralement, l'organisation du cours constituent les facteurs explicatifs les plus importants du taux de rétention: " the more satisfied (i.e. positive sentiment) a student is with the professor, the teaching material, and the assignments, the more probable that s/he will successfully complete the course " (2013, p. 11). Dans notre cas, $31 \%$ des répondants à l'enquête affirment s'être sentis en relation avec le(s) professeur(s) pendant le cours alors qu'ils sont près de $90 \%$ à avoir apprécié l'animation et l'encadrement du MOOC par l'équipe pédagogique, et plus de $90 \%$ à avoir apprécié la conception pédagogique des leçons. Parmi les facteurs ayant motivé le journal Le Monde à faire figurer le MOOC Louv3x, qui plus est, en première place, dans sa sélection des dix MOOC « incontournables de la rentrée 2014 » (Paoli, 2014), la question se pose de savoir quels seraient les facteurs influençant une telle estimation. Plus que les modalités d'un cours, il semble que ce soit sa qualité qui influence l'apprentissage des étudiants, alors même que la construction et l'animation d'un cours en ligne de qualité, offrant suffisamment de soutien aux étudiants, se révèlent plus difficiles que pour des cours traditionnels (Xu et Jaggars, 2011, p. 375). Sur la base d'un tel constat, nous proposons de distinguer, d'une part, la dimension technique et, d'autre part, la dimension pédagogique du MOOC Louv3x.
Celui-ci a été développé sur la plateforme edX, une initiative de l'Université Harvard et du Massachusetts Institute of Technology (MIT) gérée par une équipe interdisciplinaire d'environ 150 employés, en poste aux États-Unis, et dont l'ambition est d'offrir aux équipes enseignantes des établissements partenaires situés dans les différents pays un environnement favorable pour le développement de MOOC. Comme le note El-Hmoudova (2014, p. 31) : "For universities, MOOCs offer the great potential for building and extending the university as a brand. Harvard and MIT use their edX format to showcase their world-class offerings, and the networked nature of the platform expands the reach of their courses to partners and students who might never otherwise have access. ». Cependant, comme le dispositif technique - design de l'interface, ergonomie, expérience utilisateur - proposé est similaire pour l'ensemble des MOOC edX, il ne constitue pas un facteur discriminant pour analyser des taux de rétention différenciés entre les cours développés sur cette même plateforme.

Outre la dimension technique, la pédagogie centrée sur les apprenants dans une approche constructiviste (que nous détaillerons ci-dessous) encourage leur engagement (Toven-Lindsey et al., 2015, p. 1) et c'est donc une dimension que nous retenons. Louv3x repose sur une équipe enseignante constituée de professeurs d'université et d'assistants en science politique, de techniciens spécialisés en supports audiovisuels et Internet, ainsi que de conseillers pédagogiques attachés à l'Université catholique de Louvain (UCL). La littérature recense la présence de «community TAs» (ou «teaching assistants ») comme un facteur accroissant la motivation des apprenants et le taux de réussite, car ils viennent à l'appui des professeurs pour assurer l'interactivité, plus importante dans un cours massif en ligne que dans un cours traditionnel (Khalil et Ebner, 2014, p. 1241). C'est également en concertation avec l'Institut de pédagogie universitaire et des multimédias (IPM), expert, au sein de l'UCL, de la mise en place de dispositifs pédagogiques et notamment des MOOC, que des fondements de l'apprentissage ont été soumis à des 
délibérations et à des décisions, comme, par exemple, la formulation des objectifs du cours et des compétences ("learning outcomes») à acquérir par les apprenants, ou encore les effets rétroactifs $\mathrm{du}$ MOOC sur les pratiques des professeurs. La coopération coordonnée et permanente au sein de l'équipe et avec son environnement a impliqué, en amont du démarrage en ligne, des "MOOC testeurs » aux profils différenciés (en termes de sexe, d'âge, de profession) et des étudiants en science politique. D'une part, ils ont fourni des rétroactions permettant des ajustements où cela s'avérait nécessaire et, d'autre part, ils ont, plus spécifiquement, partagé avec l'équipe leurs visions sur la chose politique et le contenu du MOOC. Situer le MOOC dans un cycle d'amélioration continue sur la base des rétroactions, discussions et réunions entre tous les acteurs du MOOC est un objectif omniprésent de l'équipe. Ainsi, les commentaires d'apprenants sur le forum général qui permettent d'améliorer la structure technique et la dynamique pédagogique du cours sont régulièrement pris en considération à l'occasion des réunions d'équipe.

\subsection{La structure réflexive du cours}

Pour sa première édition, Louv3x s'étend sur une période totale de six semaines. Toutes les semaines du MOOC comportent principalement pour les apprenants un apprentissage théorique et des exercices d'application (notamment liés à l'actualité) sur les thèmes et/ou notions suivants : "Qu'est-ce que la science politique? », « L'État », « La démocratie », «Les idéologies» et «Le pouvoir ». La dernière semaine est consacrée au test final sur l'ensemble de la matière. Le MOOC offre en outre une introduction, ainsi qu'une conclusion générales.

En termes d'activités par les apprenants, un forum général accompagne chaque thème afin de permettre à ceux-ci de poser des questions et d'interagir entre eux et avec l'équipe pédagogique. Une page reprenant des références bibliographiques étaye le contenu des vidéos et permet aux apprenants d'aller plus loin dans leur apprentissage. Afin de renforcer la dimension interactive du cours, une «Synthèse de la semaine » est offerte aux apprenants dans le cadre de Google Hangouts hebdomadaires d'une heure. Lors de ceux-ci, les professeurs répondent aux questions posées en ligne ou postées sur les forums. La vidéo peut être suivie en direct ou en différé. L'objectif de cette démarche est de renforcer l'interaction avec les apprenants et l'ancrage du cours dans un apprentissage en continu.

Pour la semaine sur « Le pouvoir », un accent plus pratique a été imprimé. Concrètement, l'équipe pédagogique a réalisé, puis posté, des entretiens avec quatre acteurs de terrain confrontés au pouvoir dans leur pratique quotidienne à l'échelon européen : une femme politique, une syndicaliste, un journaliste et un lobbyiste. Ayant visionné les vidéos de leçons théoriques et d'entretiens, les apprenants ont été invités à aller eux-mêmes rencontrer sur le terrain des acteurs confrontés au pouvoir et à en réaliser une interview afin de pouvoir en débattre avec les autres apprenants. Un volet méthodologique sur la technique d'entretien dans une démarche scientifique et sur la réalisation d'une vidéo a été inclus dans le MOOC à l'intention des apprenants.

Si l'on se réfère au schéma d'analyse d'Arbaugh et Benbunan-Fich (2006) relatif aux approches d'enseignement, il est possible de distinguer des expériences d'enseignement objectivistes-individuelles (les apprenants reçoivent la matière de l'équipe pédagogique selon le design et le rythme choisis par cette dernière), objectivistes-collectives (les apprenants déploient des stratégies collaboratives dans l'optique d'une communauté d'apprenants), constructivistes-individuelles (les apprenants constituent leur savoir par leurs interactions personnelles avec la matière) et constructivistes-collectives (les apprenants interagissent les uns avec les autres dans la co-construction d'un savoir). Dans son approche, le MOOC Louv3x combine des dimensions objectivistes-individuelles (transfert d'informations par des leçons-vidéos), objectivistes-collectives (forum de discussion en ligne), des dimensions constructivistes-individuelles (réalisation d'une courte capsule vidéo par chaque apprenant sur le thème du pouvoir), voire des dimensions constructivistes-collectives (même s'il s'est révélé 
difficile d'associer les apprenants aux séances hebdomadaires de questions-réponses en direct via le support Google Hangout).

En termes d'approche objectiviste-individuelle, les répondants à l'enquête indiquent, en général, une appréciation positive de la charge de travail, du calendrier ainsi que de la tonalité du cours, même si $20 \%$ d'entre eux ont apprécié négativement la charge de travail et le calendrier. Notons que $70 \%$ des répondants affirment s'être agréablement sentis guidés et avoir su ce qu'ils devaient faire exactement. Les apprenants étaient encouragés à développer des stratégies de coopération entre eux, ce qui a été exprimé par $25 \%$ des répondants, qui estiment avoir eu le sentiment d'être en relation avec d'autres apprenants du cours. Enfin, il est à remarquer que $90 \%$ des apprenants ayant participé à l'enquête affirment avoir étudié seuls et principalement chez eux.

Peu de MOOC à l'heure actuelle sont répertoriés qui combinent des activités pédagogiques répondant à des dynamiques plurielles d'appropriation du savoir et de communication sur lui (Toven-Lindsey et al., 2015, p. 7). Or la combinaison de modes opératoires variés pour la transmission des connaissances et leur discussion critique peut contribuer à améliorer l'engagement et donc le taux de réussite des apprenants.

Le tableau I recense des exemples d'activités du MOOC Louv3x selon l'approche dans laquelle elles s'inscrivent principalement. Certaines de ces activités sont «obligatoires » alors que d'autres sont facultatives, permettant ainsi aux apprenants d'aller plus loin, par exemple, au travers de lectures complémentaires. L'engagement des apprenants est donc à géométrie variable. Mais dans l'optique « keeping them clicking », pour reprendre l'expression de Cassidy et al. (2014), l'engagement des apprenants a été lié à leur motivation de se voir progresser dans le cours. En participant aux diverses activités et en cliquant sur leur validation, les apprenants augmentaient leur taux vers la certification à $75 \%$, dont nous allons détailler l'évaluation dans la section suivante.
Tableau I: Les modes opératoires variés de Louv3x selon le schéma d'Arbaugh et Benbunan-Fich (2006)

\begin{tabular}{|c|c|c|c|}
\hline & \multicolumn{2}{|c|}{ Dimension sociale } \\
\hline & & individuelle & collective \\
\hline \multirow[b]{2}{*}{$\begin{array}{l}\text { Dimension épis- } \\
\text { témologique }\end{array}$} & objectiviste & $\begin{array}{l}\text { Visionner une vi- } \\
\text { déo sur la notion } \\
\text { de paradigme et } \\
\text { sur l'évolution } \\
\text { des frontières à } \\
\text { travers le temps }\end{array}$ & $\begin{array}{l}\text { Poster une affi- } \\
\text { che électorale } \\
\text { qui illustre une } \\
\text { idéologie et } \\
\text { son rapport } \\
\text { au pouvoir, la } \\
\text { commenter } \\
\text { pour les autres } \\
\text { apprenants, } \\
\text { discuter les } \\
\text { images postées } \\
\text { par les autres } \\
\text { apprenants }\end{array}$ \\
\hline & constructiviste & $\begin{array}{l}\text { - Alimenter } \\
\text { un nuage de } \\
\text { mots autour } \\
\text { de la notion } \\
\text { «politique » } \\
\text { - Contribuer à un } \\
\text { wiki selon son } \\
\text { pays d'origine } \\
\text { pour échanger } \\
\text { sur la question: } \\
\text { "Comment } \\
\text { votre État est-il } \\
\text { organisé?» }\end{array}$ & $\begin{array}{l}\text { - Lire des } \\
\text { extraits } \\
\text { (commen- } \\
\text { tés par les } \\
\text { professeurs) } \\
\text { du Prince de } \\
\text { Machiavel } \\
\text { pour discuter } \\
\text { ensemble } \\
\text { dans une } \\
\text { perspec- } \\
\text { tive critique la } \\
\text { question } \\
\text { «La fin justi- } \\
\text { fie-t-elle les } \\
\text { moyens? » } \\
\text { - Réaliser un } \\
\text { entretien } \\
\text { filmé (capsule } \\
\text { vidéo), le pos- } \\
\text { ter, réagir aux } \\
\text { commentaires }\end{array}$ \\
\hline
\end{tabular}




\subsection{L'évaluation de l'apprentissage}

L'évaluation des apprenants dans le cadre du MOOC Louv3x est divisée en trois grands volets. Le premier volet est celui de la « participation active » (PA) qui vise véritablement à encourager l'investissement des apprenants tout au long du cours, sans recourir à une évaluation directement certificative. En effet, $50 \%$ du résultat final est octroyé sur la base de l'achèvement d'activités, dont le visionnage de vidéos. Comment cette PA est-elle mesurée? Dans un premier temps, un point est attribué au visionnage de chaque vidéo et un autre à la réponse correcte au «quiz d'apprentissage ». En outre, toute participation à une activité rapporte également des points. Dès lors, un suivi actif du cours octroie à l'apprenant la moitié des points nécessaires pour la réussite du MOOC. La PA est accessible pendant l'entièreté du cours; cela signifie concrètement qu'un apprenant n'ayant pas eu le temps de travailler une semaine peut toujours y retourner par la suite et valoriser sa participation. Le MOOC a ainsi agi sur deux contraintes évoquées dans la littérature concernant les apprenants : la baisse de motivation et le manque de temps (Bonk et Khoo, 2014, p. 25; Khalil et Ebner, 2014, p. 1239; Onah et al., 2014, p. 5828; Zheng et al., 2015, p. 8).

Le deuxième volet de l'évaluation porte sur des examens partiels. Après chaque semaine (sauf pour la dernière), un questionnaire à choix multiples (QCM) de cinq questions, relatives à l'apprentissage de la semaine, est soumis aux participants du cours. La finalité du MOOC étant la découverte d'une discipline à travers une diversité de modes opératoires en équilibrant la gestion du temps, les tests hebdomadaires ont comporté un nombre limité de questions. Ils s'ajoutent à ceux qui accompagnent chaque leçon. De plus, la démarche étant pédagogiquement conçue comme certificative, mais aussi formative, les participants ont la possibilité de lire la question de l'examen partiel, et - s'ils en ont besoin - de visionner à nouveau la vidéo avant de répondre. Les réponses correctes sont valorisées à hauteur de $25 \%$ du score final pour l'ensemble du MOOC.
Le troisième et dernier volet évaluatif se compose d'un examen final : 20 questions fermées abordent toute la matière du cours dans un ordre aléatoire. Le caractère massif des cours en ligne implique des modalités d'évaluation automatisées (Vallières, 2013, p. 1), mais il laisse aussi potentiellement aux apprenants une possibilité de communication entre eux pour répondre aux tests. Concernant l'automatisation des corrections, afin de renforcer la dimension pédagogique, l'examen - comme du reste tous les quizz et tests - fait suivre la correction automatisée (grâce à laquelle chaque réponse apparaît directement comme correcte ou non) d'une explication de la réponse. Concernant la communication de réponses entre apprenants, l'équipe pédagogique a posé le choix d'inscrire sa démarche dans une conception formative. La plateforme edX travaille par ailleurs davantage aux modalités de vérification des certificats qu'à celles de la réalisation individuelle d'exercices par les apprenants. Les MOOC posent aujourd'hui aux équipes pédagogiques le dilemme du contrôle et de la co-construction des savoirs.

De manière générale, le mode d'évaluation dans le MOOC Louv3x est donc axé sur la valorisation de chaque participation de l'apprenant. Certaines études ont démontré que les apprenants ne participent que très peu aux activités d'interactions, sauf si cela peut améliorer leur score final, ce qui est le cas ici (Karsenti, 2013). Effectivement, à peu d'exceptions près, toute activité sur la plateforme joue un rôle dans le score final pour l'obtention du certificat, ce qui motive les apprenants à une participation plus poussée. Cependant, cela peut entraîner des effets pervers. Ainsi, lorsque les apprenants ont par exemple l'obligation de participer à certaines discussions sur des forums, amorcées par une question (par exemple : « Êtes-vous pour ou contre l'obligation de voter? »), ils peuvent être tentés de répondre à la question brièvement et sans tenir compte des réponses déjà postées par d'autres. Ceci peut également s'expliquer par la difficulté de suivre le fil d'une discussion à laquelle participent plusieurs centaines d'apprenants. Sur le plan technique, peu de solutions sont déjà offertes pour ramifier les interventions de milliers d'apprenants. Quoi qu'il en 
soit, cela peut conduire à une situation où tout le monde parle, mais peu s'écoutent. On constate finalement que la plupart des apprenants font ce qui est demandé, ni plus ni moins. Ce constat est similaire à celui d'études déjà menées par Karsenti, Lepage et Gervais (2002).

\section{Rétention passive et rétention active}

À partir de ceci, nous proposons d'aborder une question qui se pose souvent dans la littérature concernant les MOOC, celle du choix des indicateurs pour mesurer le degré de succès d'un MOOC. Les universités utilisent souvent le nombre d'inscrits pour évaluer le succès de leurs offres de formation. Cependant, s'inscrire à un MOOC est une démarche relativement facile pour une personne ayant accès à Internet et ne l'engage pas dans un processus d'apprentissage à part entière. C'est comparable au fait, dans une librairie, de prendre un livre en mains, de le consulter, puis de le redéposer en rayon. Pour dépasser ce constat, l'équipe du MOOC Louv3x a saisi l'opportunité de collecter des données en soumettant un questionnaire aux personnes qui s'y sont inscrites afin d'évaluer le profil des participants, leur représentation du cours, leur degré d'implication, leurs attentes et les moyens utilisés pour suivre le cours.

Des 6470 invitations lancées pour répondre au questionnaire, 1593 ont permis d'alimenter l'enquête administrée au début du cours (taux de réponse : 24,6\%). À la fin du cours, 1423 des 7667 apprenants ont répondu à l'enquête administrée (taux de réponse : 18,6\%). On a pu observer une certaine attrition dans l'échantillon, car parmi les répondants, certains ont répondu à l'enquête au début et à la fin du cours alors que d'autres n'ont répondu qu'au questionnaire administré au début du MOOC (dénommé «pré»). Enfin, certains n'ont répondu qu'au questionnaire administré à la fin du cours (dénommé « post »).

Outre ces données liées aux questionnaires qui vont nourrir nos analyses, des informations liées à l'ins- cription et à la certification en tant que telles sont également accessibles et reprises au tableau II. Il en ressort qu'il est difficilement soutenable d'affirmer le succès d'un MOOC et la qualité de son apprentissage uniquement sur la base du nombre d'inscrits. Par contre, l'achèvement d'un cours est une mesure fondamentale de son succès (Xu et Jaggars, 2011, p. 361).

Tableau II : Taux de rétention active et taux de rétention passive du M00C Louv3x

\begin{tabular}{|l|c|c|}
\cline { 2 - 3 } \multicolumn{1}{c|}{} & Louv3x & Moyenne edX \\
\hline Inscrits & 7667 & \\
\hline Actifs (/nombre d'inscrits) & 1578 & $10 \%$ \\
& $(21 \%)$ & \\
\hline Certificats (/nombre d'inscrits) & 1363 & $5,80 \%$ \\
& $(18 \%)$ & \\
\hline Certificats (/nombre d'actifs) & $86 \%$ & $57 \%$ \\
\hline
\end{tabular}

Source : IPM et auteurs, 2014

N'est-il donc pas plus intéressant de mesurer la réussite d'un MOOC par le nombre d'apprenants actifs, c'est-à-dire, selon le critère défini par edX, un apprenant qui a réalisé au moins une activité de la deuxième semaine? Cela fait écho aux études qui montrent que l'attrition la plus importante se produit entre la première et la deuxième semaine d'apprentissage, suivie d'une stabilisation de l'engagement dans la suite du cours (Cassidy et al., 2014, p. 8). Par le critère d' '《 apprenant actif », tous ceux qui n'ont pas été plus loin que la première semaine ne sont pas pris en compte dans l'analyse. Par conséquent, il est impératif de créer deux indicateurs différents. En effet, la rétention des apprenants peut être calculée par le nombre d'inscrits / le nombre de certificats obtenus, ou par le nombre d'actifs / le nombre de certificats obtenus. Nonobstant des débats scientifiques autour de la notion de rétention et de son calcul (Cheung, 2014, p. 19-22), nous retenons les deux définitions suivantes. Le premier indicateur correspond au taux de réussite total, ou ce que nous appelons le taux de rétention passif. Le second correspond au taux de réussite 
parmi les apprenants actifs, que nous qualifions de taux de rétention actif. Sur la base de ces critères, le MOOC Louv3x présente une rétention passive de $18 \%$ (soit environ trois fois supérieure à la moyenne d'edX) et une rétention active de $86 \%$.

Outre les choix techniques et pédagogiques exposés ci-dessous, la rétention peut être analysée à partir du profil et des motivations des apprenants dans la mesure où ils influencent le taux d'abandon (Bonk et Khoo, 2014, p. 25; Cassidy et al., 2014, p. 13). Et l'engagement des apprenants est présenté comme leur satisfaction, leur motivation et leur persistance dans le cours (Toven-Lindsey et al., 2015 , p. 2). Il est donc important de lier le taux de rétention aux facteurs objectifs (profil type des apprenants) et subjectifs (motivations des apprenants) permettant d'expliquer un taux de succès plus élevé d'un MOOC comme c'est le cas pour Louv3x.

\subsection{L'apprenant type est un homme de 31 ans déjà diplômé}

Un des facteurs potentiellement explicatifs d'une rétention élevée est lié au profil de celui qui suit le MOOC. La répartition des apprenants en termes de sexe, d'âge, de situation géographique et de niveau du plus haut diplôme obtenu est instructive. Il convient toutefois de rappeler que certains auteurs, tel Adamopoulos, n'ont pas trouvé de lien entre la rétention et les caractéristiques des apprenants: " Finally, attrition was not found to be related with student characteristics (i.e. gender, formal education) » (2013, p. 16).

Il ressort des graphiques ci-dessous que nos apprenants se situent dans 160 pays différents, dont les trois premiers sont la Belgique, la France et les États-Unis. Ce sont majoritairement des hommes (62\%), relativement jeunes (nés dans les années 1980-1990) et ayant un diplôme d'enseignement supérieur (plus de $65 \%$ ont soit un bachelier, soit un master, soit un diplôme professionnalisant). On constate que l'âge moyen correspond pratiquement à celui de la moyenne edX qui tourne autour de la trentaine (edX, 2015).

\section{Sexe}

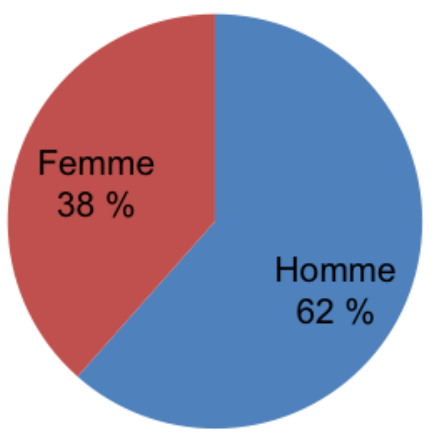

Figure 1 : Distribution des effectifs par sexe

Source : edX - 2014, à partir d'un échantillon de 7568 individus

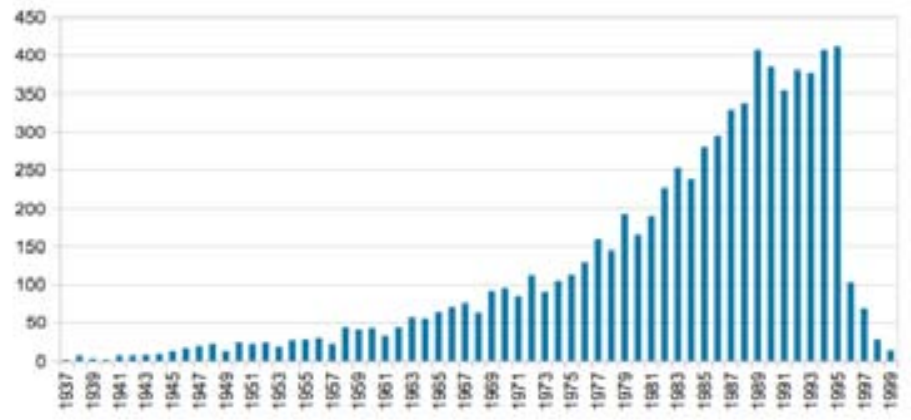

Figure 2 : Distribution des effectifs selon l'année de naissance

Source : edX - 2014, à partir d'un échantillon de 7494 individus 


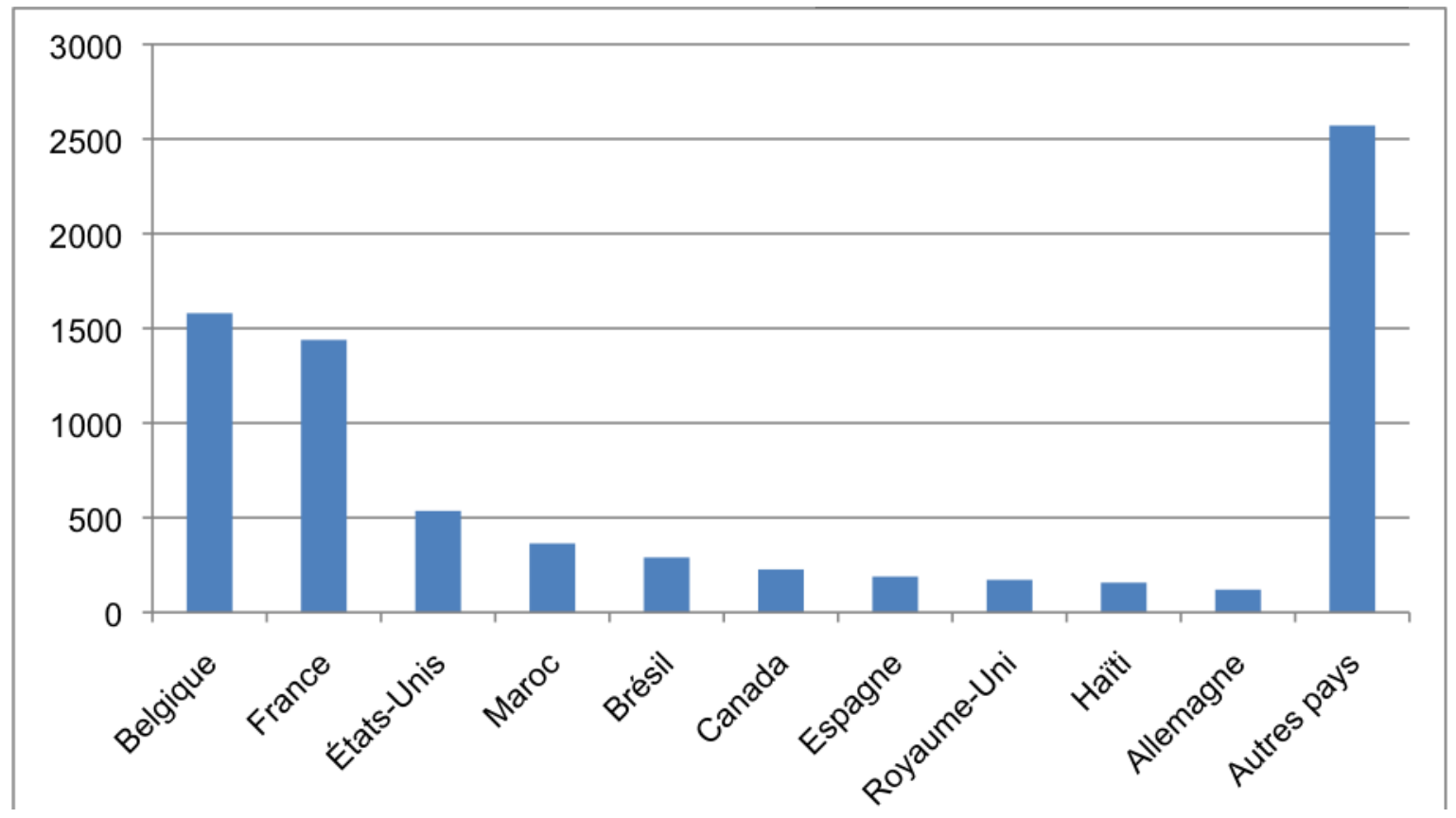

Figure 3 : Distribution des effectifs selon le pays de résidence

Source : edX - 2014, à partir d'un échantillon de 7645 individus

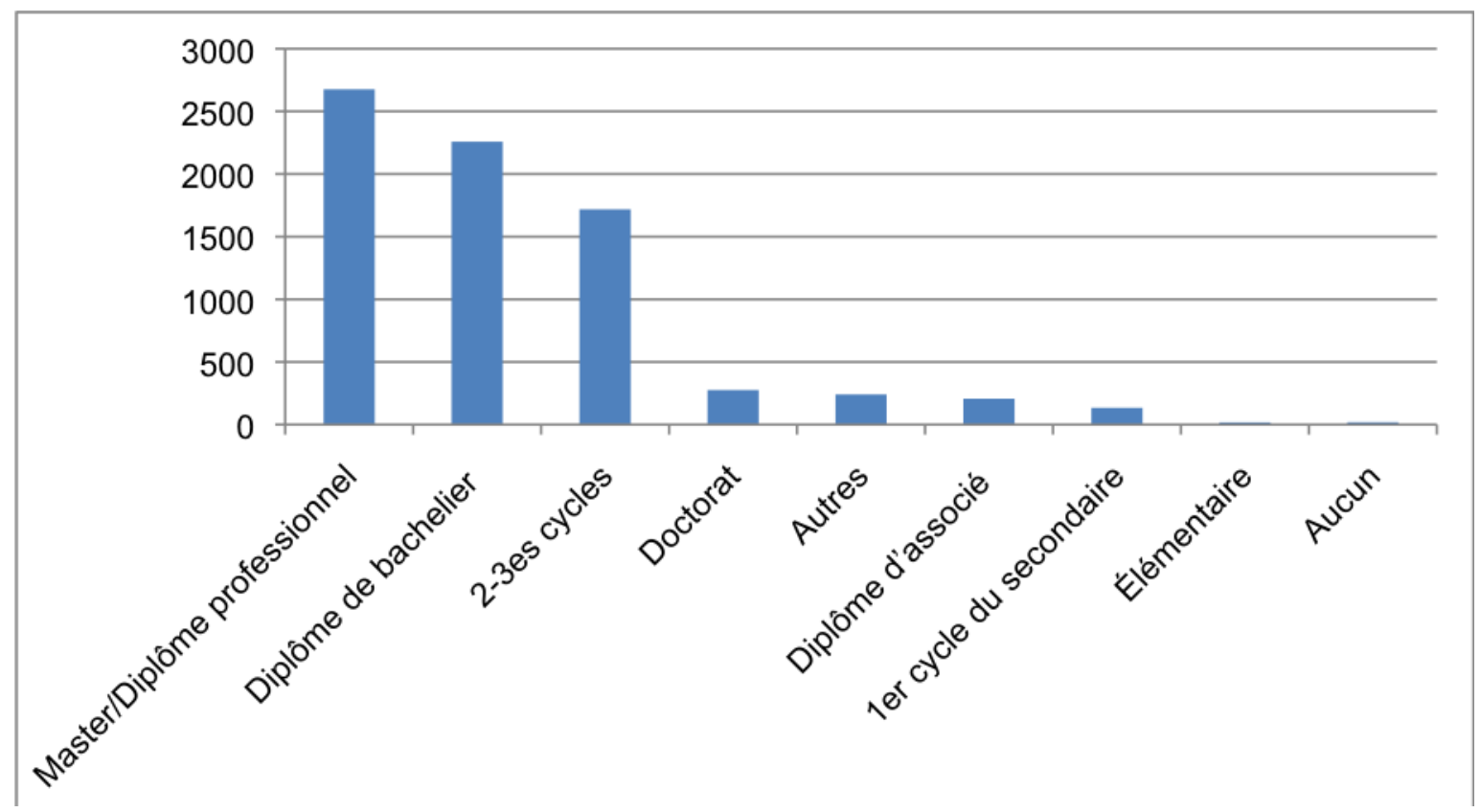

Figure 4 : Distribution des effectifs selon le plus haut niveau de diplôme obtenu

Source : edX - 2014, à partir d'un échantillon de 7547 individus 


\subsection{Les motivations d'apprentissage}

Outre les variables sociodémographiques objectives, il est essentiel de prendre en considération le poids de facteurs motivationnels et subjectifs : " [...] when we discuss retention issues in MOOCs, we must begin with a solid understanding of participants' learning goals and the implication for corresponding views of what counts as being 'finished' " (Zheng et al., 2015, p. 1892). L'intention d'obtenir le certificat se combine à une série de raisons pour lesquelles les apprenants suivent le cours jusqu'au bout, comme le montre la figure 5 relative au MOOC Louv3x.
Outre les motivations reprises à la figure 5, un certain nombre de raisons et de motivations complémentaires ont été amenées par les apprenants quand ils ont répondu à la question : « En quelques mots, pourriez-vous dire pourquoi vous vous êtes inscrit-e à ce MOOC et quelles sont vos attentes? ». Reviennent souvent l'apprentissage de la langue, les avantages spécifiques du MOOC (en ligne, flexible, interactif), ainsi que la possibilité de travailler de façon autonome et à son propre rythme. Par ailleurs, comme le montre le tableau III, on constate qu'au début et à la fin du cours, l'objectif exprimé motivant l'inscription au cours reste largement identique.

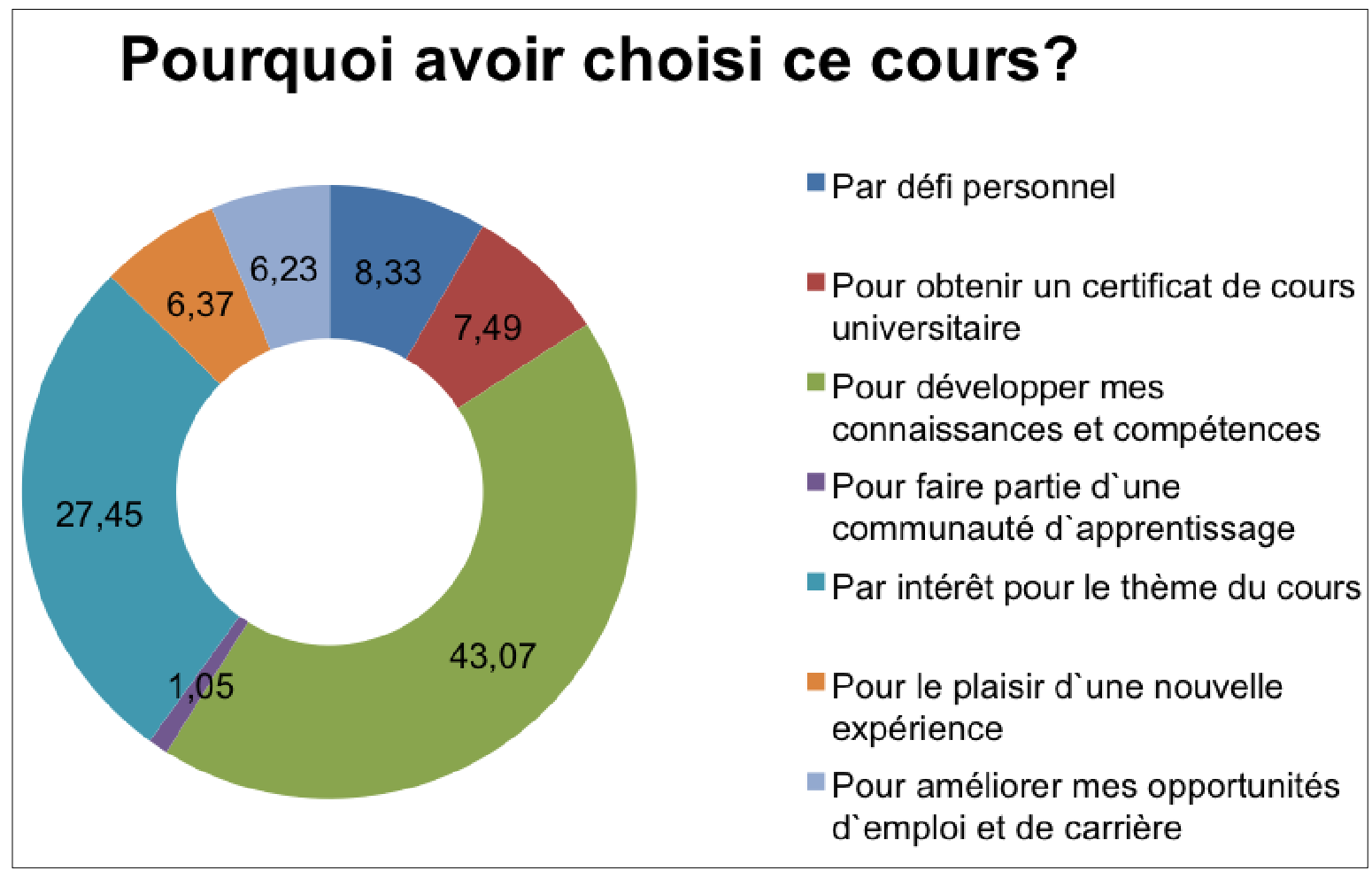

Figure 5 : Distribution des effectifs selon les raisons invoquées pour suivre le cours (pré-questionnaire, en pourcentage, à partir d'un échantillon de 1428 individus)

Source: Auteurs, 2014 - Données en pourcentage, à partir d'un échantillon de 1428 individus (pré-questionnaire); le total n'est pas égal à 100 en raison des arrondis. 
Tableau III : Distribution des avis des apprenants quant aux objectifs qu'ils avaient en s'inscrivant au cours (pré-questionnaire) / qu'ils avaient au moment de s'inscrire (post-questionnaire)

\begin{tabular}{|l|c|c|}
\hline & Pré & Post \\
\hline Juste jeter un coup d'œil. & 1,04 & 4,41 \\
\hline $\begin{array}{l}\text { Je vais faire seulement ce qui } \\
\text { m'intéresse, sans doute pas } \\
\text { tout le cours. }\end{array}$ & 4,73 & 3,40 \\
\hline $\begin{array}{l}\text { J'envisage de suivre l'ensemble } \\
\text { du cours de façon passive, sans } \\
\text { participer aux activités. }\end{array}$ & 9,87 & 14,23 \\
\hline $\begin{array}{l}\text { Je pense réaliser toutes les } \\
\text { activités, mais je ne me mets } \\
\text { pas la pression pour cela. }\end{array}$ & 39,19 & 33,87 \\
\hline $\begin{array}{l}\text { J'ai la ferme intention de } \\
\text { réaliser toutes les activités et } \\
\text { d'obtenir le certificat. }\end{array}$ & 45,17 & 44,08 \\
\hline
\end{tabular}

Source: Auteurs, 2014 - Données en pourcentage, à partir d'un échantillon de 1439 individus (pré-questionnaire) et de 1293 individus (post-questionnaire); le total n'est pas toujours égal à 100 en raison des arrondis.

De manière générale, on peut remarquer que plus de $40 \%$ des répondants affirment avoir la ferme intention de réaliser toutes les activités et d'obtenir le certificat. À ce groupe s'ajoutent un peu moins de $40 \%$ de répondants souhaitant réaliser toutes les activités, sans se mettre de pression pour cela. Au total, on constate que plus de $84 \%$ des répondants indiquent dans le pré-questionnaire qu'ils souhaitent achever le MOOC. Au terme du MOOC (postquestionnaire), ils sont $78 \%$ à avoir déclaré qu'ils avaient l'intention de réaliser toutes les activités, sans pression ou avec l'intention d'être certifié. Pour rappel, $86 \%$ des apprenants actifs ont finalement décroché le certificat.

\subsection{Les attentes par rapport aux acquis d'apprentissage et leur évolution}

Pour mieux cerner les attentes d'apprentissage des apprenants, nous leur avons demandé lors du démarrage du cours dans quelle mesure ils pensaient être capables à la fin du MOOC : a) de comprendre, définir et expliquer des concepts en science politique; b) de mettre en lien les concepts avec leurs auteurs et, plus largement, les phénomènes concrets qu'ils désignent, et, finalement; c) d'amorcer des analyses objectives de phénomènes politiques basées sur les connaissances acquises. Les apprenants avaient le choix, sur une échelle de Likert, entre cinq modalités allant de «Pas du tout d'accord» à « Tout à fait d'accord».

Tableau IV : Distribution des effectifs selon les attentes d'apprentissage estimées avant le démarrage du MOOC

\begin{tabular}{|l|c|c|c|}
\hline Pré & $\begin{array}{c}\text { Comprendre, dé- } \\
\text { finir et expliquer } \\
\text { des concepts }\end{array}$ & $\begin{array}{c}\text { Mettre en } \\
\text { lien des } \\
\text { concepts }\end{array}$ & $\begin{array}{c}\text { Amorcer des } \\
\text { analyses } \\
\text { objectives }\end{array}$ \\
\hline $\begin{array}{l}\text { Pas du tout } \\
\text { d'accord }\end{array}$ & 1,84 & 1,30 & 1,78 \\
\hline $\begin{array}{l}\text { Plutôt pas } \\
\text { d'accord }\end{array}$ & 1,78 & 2,60 & 1,98 \\
\hline $\begin{array}{l}\text { Ni d'accord ni pas } \\
\text { d'accord }\end{array}$ & 2,39 & 9,43 & 5,87 \\
\hline Plutôt d'accord & 23,98 & 40,51 & 32,45 \\
\hline $\begin{array}{l}\text { Tout à fait } \\
\text { d'accord }\end{array}$ & 70,01 & 46,17 & 57,92 \\
\hline N & \multicolumn{2}{|l}{1464} \\
\hline
\end{tabular}

Source: Auteurs, 2014 - Données en pourcentage, à partir d'un échantillon de 1464 individus (pré-questionnaire); le total n'est pas toujours égal à 100 en raison des arrondis.

Le tableau IV révèle que les attentes par rapport aux objectifs d'apprentissage sont élevées: environ $95 \%$ des apprenants ayant répondu au préquestionnaire estiment qu'ils seront capables à la fin du MOOC de comprendre, définir et expliquer des concepts. La même tendance s'observe pour les deux autres objectifs d'apprentissage (respectivement environ $87 \%$ et $90 \%$ ). 
Les mêmes questions ont été posées à la fin du MOOC dans le post-questionnaire, les répondants devant cette fois répondre sur une échelle graduée de 1 à 10 , de sorte que nous puissions obtenir des résultats plus précis. Ici, deux questions leur étaient posées. La première concerne leur compétence avant le cours : " Où se situe votre degré de compétence sur ces objectifs avant que le cours commence? » Les trois objectifs sont « définir et expliquer les concepts en science politique », «mettre en lien les auteurs et, plus largement, les phénomènes concrets qu'ils désignent » et enfin, « amorcer des analyses objectives de phénomènes politiques basées sur les connaissances acquises ». La seconde concerne leur compétence après le cours : «Et maintenant que le cours est terminé, comment estimez-vous votre degré de compétence par rapport à ces objectifs? » Les trois figures suivantes montrent la distribution de l'évolution de positionnement des apprenants avant et après.

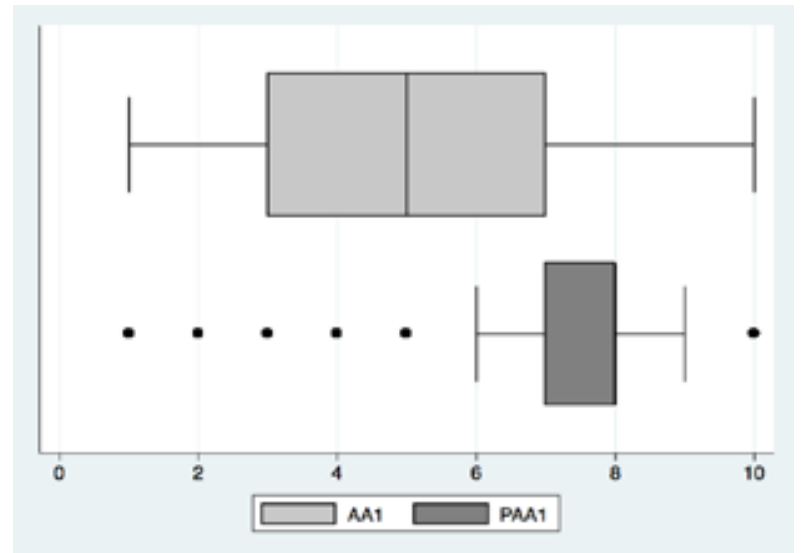

Légende $: \mathrm{AA} 1=$ objectif avant le $\mathrm{MOOC} ; \mathrm{PAA} 1=$ objectif après le MOOC.

Figure 6 : Définir et expliquer les concepts en science politique

Source : Auteurs, 2014, à partir d'un échantillon de 1207 individus (post-questionnaire).

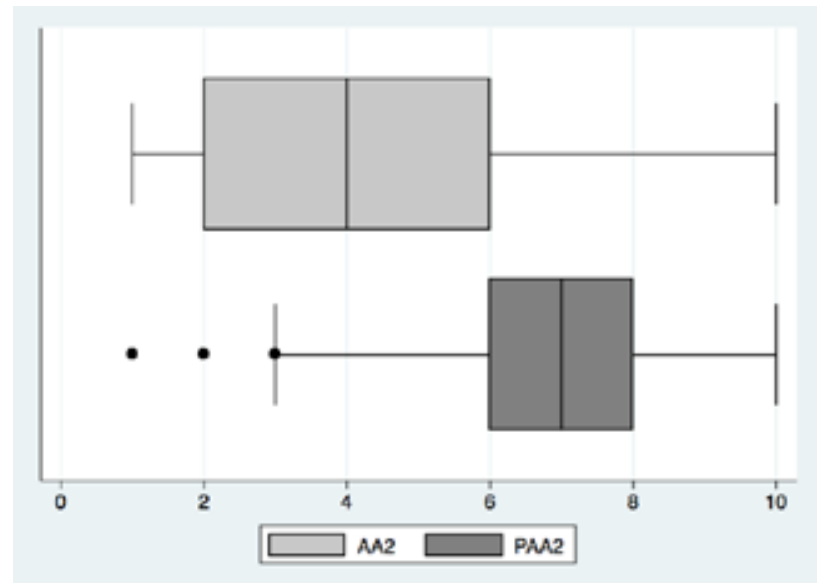

Légende : $\mathrm{AA} 2=$ objectif avant le MOOC; PAA2 = objectif après le MOOC.

Figure 7: Mettre en lien les auteurs et, plus largement, les phénomènes concrets qu'ils désignent

Source : Auteurs, 2014, à partir d'un échantillon de 1207 individus (post-questionnaire).

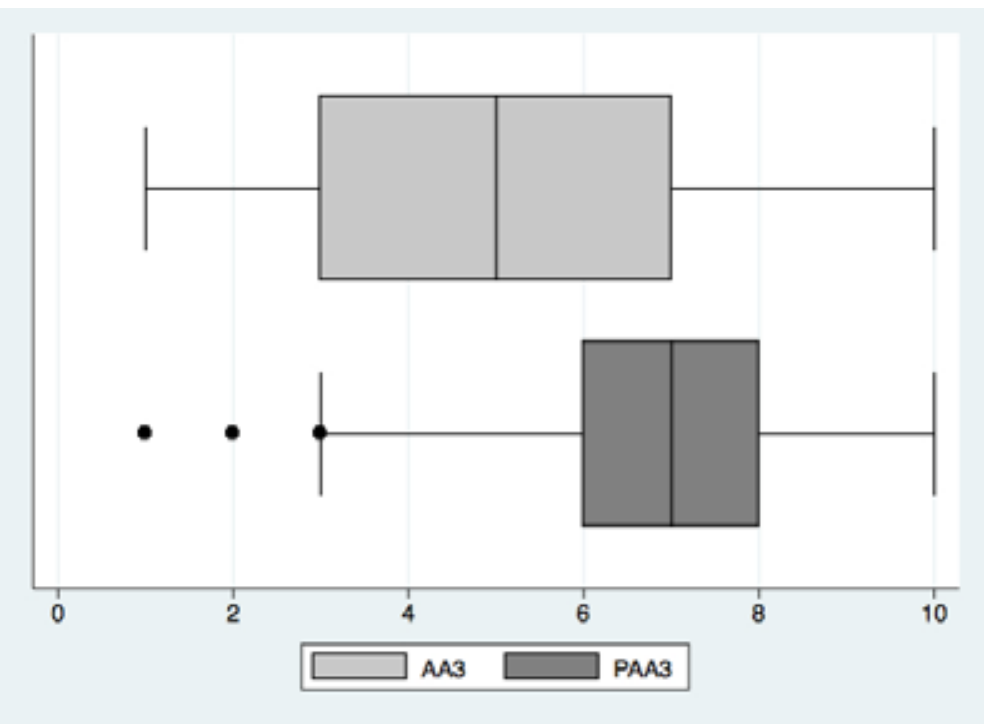

Légende : AA3 = objectif avant le MOOC; PAA3 = objectif après le MOOC.

Figure 8: Mettre en lien les auteurs et, plus largement, les phénomènes concrets qu'ils désignent

Source : Auteurs, 2014, à partir d'un échantillon de 1207 individus (post-questionnaire). 
L'évaluation axée sur la récompense a sûrement poussé les apprenants à accroître leur participation ainsi qu'à suivre le MOOC jusqu'à son terme. L'interactivité forte durant toute la première édition du cours et le sentiment d'appartenir à une communauté d'apprenants convergent également vers les constats de la littérature (Khalil et Ebner, 2014; Zheng et al., 2015). Nos données confirment des tendances bien établies, comme le fait d'avoir une préparation antérieure à l'engagement dans un cours en ligne pour augmenter son taux de réussite (Xu et Jaggars, 2011, p. 362). L'article contribue à mieux connaître la relation entre l'engagement, la rétention (dont il a proposé une nouvelle conceptualisation, en termes de « rétention active» vs. rétention "passive», ainsi que son opérationnalisation) et les motivations, à la fois des apprenants et de l'équipe pédagogique qui seraient, pour Adamopoulos (2013), le facteur influençant le plus la rétention. L'analyse des motivations et des attentes par rapport aux acquis d'apprentissage indique qu'il existe un lien entre les objectifs que se fixent les participants, leurs attentes, et la représentation de ce qu'ils pensent en retirer, notamment en termes de compétence.

Ces motivations et attentes influencent également la rétention. En effet, plus l'objectif fixé par l'apprenant lui-même, avant le cours, est élevé (c'està-dire la volonté d'obtenir le certificat), plus les probabilités sont grandes qu'il réalise l'ensemble des activités et ainsi obtienne ce certificat. Mais il ne s'agit pas uniquement d'une simple question de volonté, car cette relation doit se comprendre dans la multidimensionnalité qu'offre l'environnement d'un MOOC. La structure du MOOC, en ce qu'elle rend possible des expériences d'enseignement différentes (Arbaugh et Benbunan-Fich, 2006), offre un environnement d'apprentissage multiple, ce qui peut aider les apprenants à rencontrer les objectifs en termes de contenu et de méthode, tout en veillant à garder leur motivation élevée. Ainsi, le MOOC Louv3x, en proposant des expériences d'enseignement objectivistes-individuelles, objectivistes-collectives, constructivistes-individuelles et, dans une moindre mesure, constructivistes-collectives, a pu contribuer à des dynamiques plurielles d'appropriation du savoir qui ont elles-mêmes maintenu la motivation des apprenants, ce qui explique en partie son taux de rétention, tant passif qu'actif, supérieur à la moyenne des MOOC.

\section{Références}

Adamopoulos, P. (2013). What makes a great MOOC? An interdisciplinary analysis of student retention in online courses. Communication présentée à la Thirty Fourth International Conference on Information Systems, Milan. Récupéré du site de l'association WICHE Cooperative for Educational Technologies : http://wcet.wiche.edu

Allen, E. et Seaman, J. (2013). Changing course: Ten years of tracking online education in the United States. Récupéré de http://onlinelearningconsortium.org

Anderson, T. (2013). Promise and/or peril: MOOCs and open and distance education. Université d'Athabasca. Récupéré du site de l'organisme Commonwealth of Learning : http://www.col.org

Arbaugh, J. B. et Benbunan-Fich, R. (2006). An investigation of epistemological and social dimensions of teaching in online learning environments. The Academy of Management Learning and Education, 5(4), 435-447. doi:10.5465/AMLE.2006.23473204

Bonk, C. J. et Khoo, E. (2014). Adding some TEC-VARIETY: 100+ activities for motivating and retaining learners online. Bloomington, IN : Open World Books.

Cassidy, D., Breakwell, N. et Bailey, J. (2014). Keeping them clicking: Promoting student engagement in MOOC design. The All Ireland Journal of Teaching and Learning in Higher Education, 6(2), 1-15. Récupéré du site de l'International conference on engaging pedagogy : http://icep.ie/

Cheung, E. (2014). Analyzing student engagement and retention in Georgetown's first MOOC: Globalization's winners and losers: Challenges for developed and developing countries (mémoire de maîtrise, Université de Georgetown, Washington, DC). Récupéré du dépôt DigitalGeorgetown : http://repository.library.georgetown.edu 
Cormier, D. et Gillis, N. (2010). What is a MOOC? [vidéo]. Récupéré de http://www.youtube.com

Dillenbourg, P., Fox, A., Kirchner, C., Mitchell, J. et Wirsing, M. (2014). Massive open online courses: Current state and perspectives. Dagstuhl Manifestos, 4(1), 1-27. Récupéré du site de l'événement Ethical Forum : http://ethicalforum2013.fuus.be/

edX. (2015). Media kit. Récupéré en 2015 de http://www.edx.org

El-Hmoudova, D. (2014). MOOCs motivation and communication in the cyber learning environment. Procedia - Social and Behavioral Sciences, 131, 29-34. doi:10.1016/j.sbspro.2014.04.074

Fairhurst, A. M. et Fairhurst, L. L. (1995). Effective teaching, effective learning: making the personality connexion in the classroom. Palo Alto, CA : Davies-Black.

Gaebel, M. (2013). MOOCs - Massive open online courses - EUA Occasional Papers. Récupéré du site de l'European university association : http://www.eua.be

Giroux, H. A. (2011). On critical pedagogy. New York, NY : Continuum International Publishing Group.

Hew, K. F. et Cheung, W. S. (2014). Students' and instructors' use of massive open online courses (MOOCs): Motivations and challenges. Educational Research Review, 12, 45-58. doi:10.1016/j.edurev.2014.05.001

Karsenti, T. (2013). MOOC : révolution ou simple effet de mode? Revue internationale des technologies en pédagogie universitaire, 10(2), 6-22. Récupéré de http://www.ritpu.org

Karsenti, T., Lepage, M. et Gervais, C. (2002). @ccompagnement des stagiaires à l'ère des TIC : forum électronique ou groupe de discussion? Formation et profession, 8(2), 7-12. Récupéré de http://crifpe.ca

Khalil, H. et Ebner, M. (2014). MOOCs completion rates and possible methods to improve retention - A literature review. Dans Proceedings of World Conference on Educational Multimedia, Hypermedia and Telecommunications 2014 (p. 1236-1244). Chesapeake, VA : AACE. Récupéré de http://www.researchgate.net
Onah, D. F. O., Sinclair, J. et Boyatt, R. (2014). Dropout rates of massive open online courses: Behavioural patterns. Dans EDULEARN14 Proceedings (p. 5825-5834). Barcelone, Espagne. Récupéré de Warwick Research Archive Portal : http://wrap.warwick.ac.uk

Paoli, E. (2014, 27 août). Les MOOC incontournables de la rentrée. Le Monde. Récupéré de http://www.lemonde.fr

Pappano, L. (2012, 2 novembre). The year of the MOOC. The New York Times, p. ED26. Récupéré de http://nytimes.com

Toven-Lindsey, B., Rhoads, R. A. et Berdan Lozano, J. (2015). Virtually unlimited classrooms: Pedagogical practices in massive open online courses. Internet and Higher Education, 24, 1-12. doi:10.1016/ j.iheduc.2014.07.001 Récupéré de http://drive.google.com

Vallières, C. (2013). L'évaluation des apprentissages : un défi à l'échelle des MOOC. Perspectives SSF, (janvier 2013). Récupéré de http://www.usherbrooke.ca

Xu, D. et Jaggars, S. S. (2011). The effectiveness of distance education across Virginia's community colleges: Evidence from introductory college-level math and English courses. Educational Evaluation and Policy Analysis, 33(3), 360-377. doi:10.3102/0 162373711413814

Zheng, S., Rosson, M. B., Shih, P. C. et Carroll, J. M. (2015). Understanding student motivation, behaviors, and perceptions in MOOCs. CSCW'15 Proceedings of the 18th ACM Conference on Computer Supported Cooperative Work \& Social Computing (p. 1882-1895). Récupéré de http://www.researchgate.net 